Article

\title{
A Novel Methodology for the Synchronous Collection and Multimodal Visualization of Continuous Neurocardiovascular and Neuromuscular Physiological Data in Adults with Long COVID
}

\author{
Feng Xue ${ }^{1,2, * \mathbb{D}}$, Ann Monaghan ${ }^{1,2}$, Glenn Jennings ${ }^{1,2} \mathbb{D}$, Lisa Byrne ${ }^{3}$, Tim Foran ${ }^{4}$, Eoin Duggan ${ }^{1,2,3}$ \\ and Roman Romero-Ortuno $1,2,3,5$ (D)
}

1 Discipline of Medical Gerontology, School of Medicine, Trinity College Dublin, D02PN40 Dublin, Ireland; ann.monaghan@tcd.ie (A.M.); gljennin@tcd.ie (G.J.); dugganeo@tcd.ie (E.D.); romeroor@tcd.ie (R.R.-O.)

2 The Irish Longitudinal Study on Ageing, Trinity College Dublin, D02R590 Dublin, Ireland

3 Falls and Syncope Unit, Mercer's Institute for Successful Ageing, St. James's Hospital, D08E191 Dublin, Ireland; libyrne@stjames.ie

4 Department of Medical Physics and Bioengineering, Mercer's Institute for Successful Ageing, St. James's Hospital, D08E191 Dublin, Ireland; tforan@tcd.ie

5 Global Brain Health Institute, Trinity College Dublin, D02PN40 Dublin, Ireland

* Correspondence: fxue@tcd.ie; Tel.: +353-1-428-4614; Fax: +353-1-896-3407

check for updates

Citation: Xue, F.; Monaghan, A.; Jennings, G.; Byrne, L.; Foran, T.; Duggan, E.; Romero-Ortuno, R. A Novel Methodology for the Synchronous Collection and Multimodal Visualization of Continuous Neurocardiovascular and Neuromuscular Physiological Data in Adults with Long COVID. Sensors 2022, 22, 1758. https:// doi.org/10.3390/s22051758

Academic Editor: Andrés Ortiz García

Received: 18 January 2022

Accepted: 16 February 2022

Published: 24 February 2022

Publisher's Note: MDPI stays neutral with regard to jurisdictional claims in published maps and institutional affiliations.

Copyright: (C) 2022 by the authors. Licensee MDPI, Basel, Switzerland. This article is an open access article distributed under the terms and conditions of the Creative Commons Attribution (CC BY) license (https:// creativecommons.org/licenses/by/ $4.0 /)$.

\begin{abstract}
Background: Reports suggest that adults with post-COVID-19 syndrome or long COVID may be affected by orthostatic intolerance syndromes, with autonomic nervous system dysfunction as a possible causal factor of neurocardiovascular instability (NCVI). Long COVID can also manifest as prolonged fatigue, which may be linked to neuromuscular function impairment (NMFI). The current clinical assessment for NCVI monitors neurocardiovascular performance upon the application of orthostatic stressors such as an active (i.e., self-induced) stand or a passive (tilt table) standing test. Lower limb muscle contractions may be important in orthostatic recovery via the skeletal muscle pump. In this study, adults with long COVID were assessed with a protocol that, in addition to the standard NCVI tests, incorporated simultaneous lower limb muscle monitoring for NMFI assessment. Methods: To conduct such an investigation, a wide range of continuous non-invasive biomedical sensing technologies were employed, including digital artery photoplethysmography for the extraction of cardiovascular signals, near-infrared spectroscopy for the extraction of regional tissue oxygenation in brain and muscle, and electromyography for assessment of timed muscle contractions in the lower limbs. Results: With the proposed methodology described and exemplified in this paper, we were able to collect relevant physiological data for the assessment of neurocardiovascular and neuromuscular functioning. We were also able to integrate signals from a variety of instruments in a synchronized fashion and visualize the interactions between different physiological signals during the combined NCVI/NMFI assessment. Multiple counts of evidence were collected, which can capture the dynamics between skeletal muscle contractions and neurocardiovascular responses. Conclusions: The proposed methodology can offer an overview of the functioning of the neurocardiovascular and neuromuscular systems in a combined NCVI/NMFI setup and is capable of conducting comparative studies with signals from multiple participants at any given time in the assessment. This could help clinicians and researchers generate and test hypotheses based on the multimodal inspection of raw data in long COVID and other cohorts.
\end{abstract}

Keywords: continuous physiological signals; non-invasive hemodynamics; finometer; near-infrared-spectroscopy; electromyography; synchronous data collection; multimodal visualization; muscle pump; autonomic nervous system; neurocardiovascular instability; long COVID 


\section{Introduction}

The statistics from the World Health Organization [1] have been reflective of the devastating human and societal consequences that the COVID-19 pandemic has had for the past two years. Due to the swift pace in the research and development of vaccines, it has been possible to partially modify the natural course of the pandemic [2-4]. However, a prominent challenge is the emergence of the post-COVID-19 syndrome or 'long COVID' following the acute phase of the viral infection. With a plethora of debilitating symptoms across many physiological systems [5], the pathophysiology of long COVID remains elusive and subject of intense research efforts [6,7]. Adults with long COVID can be affected by orthostatic intolerance (OI) syndromes and this could be due to dysfunction of the autonomic nervous system leading to neurocardiovascular instability (NCVI) [8,9]. In addition, long COVID can also manifest with prolonged fatigue [10], which could be linked to neuromuscular function impairment (NMFI) [11,12].

NCVI refers to abnormal neural control of the cardiovascular system affecting blood pressure and heart rate behavior [13]. The current clinical NCVI assessments involve non-invasive continuous monitoring of neurocardiovascular activity (such as blood pressure, heart rate, and cerebral oxygenation levels) during orthostatic challenges, which can provide clinically useful information regarding an individual's ability to compensate and recuperate from stressors induced by orthostatic maneuvers [14]. Typical orthostatic stressors include the active (i.e., self-induced) stand or a passive (i.e., tilt table) standing test [15]. The autonomic nervous system provides the principal means to maintain homeostasis in response to orthostatic maneuvers, which humans actively perform repeatedly on a daily basis [16]. Upon transiting to an upright posture, gravity causes redistribution of blood (i.e., blood pooling) in the compliant distensible veins of the abdomen and lower extremities [17]. The consequent reduction in central venous pressure leads to a decline in venous return, stroke volume and arterial pressure [18]. The primary mechanism in response to orthostatic stress is the baroreflex mediated vagal withdrawal and activation of the sympathetic nervous system, which lead to increased heart rate and systemic vascular resistance [19]. The other compensatory mechanism for combatting an orthostatic challenge is termed the 'skeletal muscle pump'. This involves the contraction of skeletal muscles of both lower limbs and abdomen, which help maintain venous return and, consequently, cardiac output by constricting underlying veins to accelerate blood flow back to the heart [20]. While the influences of the muscle pump on hemodynamic responses have been previously investigated [21-23], NMFI assessments are not routinely added to clinical NCVI assessments. Since both the autonomic nervous system and the skeletal muscle pump could malfunction in adults previously infected by SARS-CoV-2, we hypothesized that it would be important to simultaneously assess NCVI and NMFI in the study of long COVID.

In this investigation, we hence employed a wide range of non-invasive sensing technologies, including digital artery photoplethysmography for the extraction of cardiovascular signals, near-infrared spectroscopy (NIRS) for the extraction of regional tissue oxygenation in the brain and muscle, and electromyography (EMG) for assessment of timed muscle contractions in the lower limbs, for the continuous monitoring of physiological signals to capture the characteristics of orthostatic recovery in clinical settings. Since one of the immediate challenges of this assessment was the data synchronization for cross-signal visualization, we developed and exemplified a novel technique to integrate signals from all instruments used in the assessment in a precisely synchronized fashion. Our aim was to visualize the interactions between all different physiological signals during the combined NCVI/NMFI assessment, to help researchers and clinicians generate and test hypotheses based on the multimodal inspection of raw data.

\section{Materials and Methods}

This methodological study was conducted within the wider context of a cross-sectional observational study on a cohort of 108 participants recruited for the TROPIC (Technology assisted solutions for the Recognition of Objective Physiological Indicators of post- 
Coronavirus-19 fatigue) investigation at Trinity College Dublin and St. James's Hospital Dublin, Ireland. With a mean age of 46 (range 25-78) years, the cohort consisted of 77 female and 31 male participants, $22 \%$ of whom were hospitalized in the acute phase of SARSCoV-2 infection. Details on cohort inclusion and exclusion criteria for the study have been documented elsewhere [24]. In short, the cohort included participants who had been experiencing long COVID symptoms following the acute phase of the infection. Among a series of assessments designed for the investigation, the NCVI/NMFI assessment was performed on included participants. As part of this multidisciplinary research, we set out to disseminate the methodology we created for assessing the neurocardiovascular and neuromuscular function in our long COVID participants, and the novel technique we developed for the synchronous collection and multimodal visualization of raw NCVI/NMFI signals.

\subsection{Assessment Protocol}

The NCVI assessment protocol was composed of a 3 min lying-to-standing orthostatic test (active stand), consecutively followed (after a short participant break) by a $10 \mathrm{~min}$, unmedicated 70-degree head-up tilt test. A 10 min supine rest period was required before both active stand and head-up tilt phases. For the assessment of NMFI, four $10 \mathrm{~s}$ maximum prompted muscle contractions of the thighs ('squeezes') were programmed halfway into rest periods and after both stands (Figure 1). Participants' continuous cardiovascular and neuromuscular signals were recorded for a total duration of 35-40 min, with body sensor attachments as shown in Figure 2. Termination criteria were in place to stop the assessment prematurely if any adverse hemodynamic signs or symptoms were experienced by a participant.

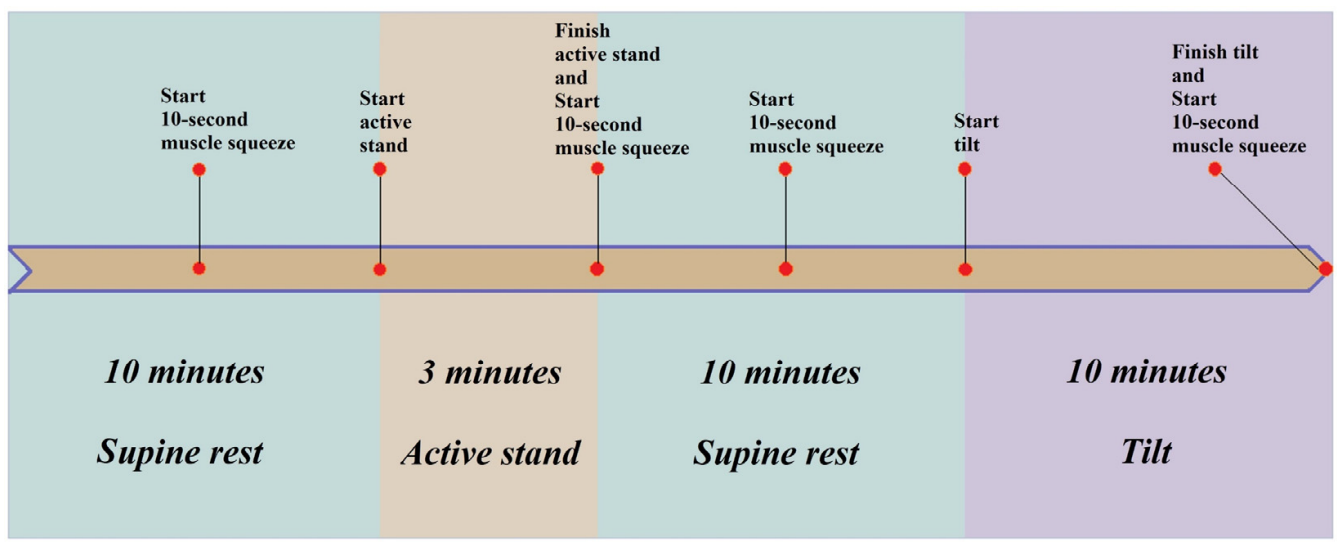

Figure 1. Diagram of the NCVI/NMFI assessment protocol. It was composed of a 10 min supine rest and a $3 \mathrm{~min}$ active stand, followed by a $10 \mathrm{~min}$ supine rest and a $10 \mathrm{~min}$ head-up tilt. With four prompted muscle contractions of the thighs introduced at different stages of the assessment, a total of six events were included (see text for further protocol details).

\subsubsection{Active Stand}

The active stand test is a clinical procedure for assessing the spectrum of abnormal cardiovascular responses to standing and evaluating causes of orthostatic intolerance, including orthostatic hypotension [25,26] and postural orthostatic tachycardia syndrome [27]. The active stand test is well established as a clinical technique for the study of orthostatic intolerance in both younger and older individuals $[15,28]$. For the active stand, participants underwent a lying-to-standing orthostatic test with non-invasive beat-to-beat blood pressure monitoring using digital photoplethysmography (Finapres ${ }^{\circledR}$ NOVA, Finapres Medical Systems, Amsterdam, The Netherlands). The height correction unit was zeroed and implemented as per the manufacturer's specifications. Participants underwent 10 min of supine rest on the horizontally leveled tilt table (Agasan KT-1060/E, AGA Sanitätsartikel GmbH, Löhne, Germany) with a height of $730 \mathrm{~mm}$. The first 1-4 $\mathrm{min}$ of the supine rest period were also used for calibration purposes, to achieve a PhysioCal repetition rate of 70 beats or 
more [29]. After at least 5 min of uninterrupted supine rest, and a $10 \mathrm{~s}$ countdown, participants were asked to stand independently and as quickly as possible. The PhysioCal, which automatically calibrates the blood pressure measured at the finger cuff, was turned off just before the stand and switched back on at 1 min post-stand. Throughout the recording, participants were asked to remain motionless and in silence with the monitored arm (left) resting extended by the side, except for reporting any symptoms of concern.

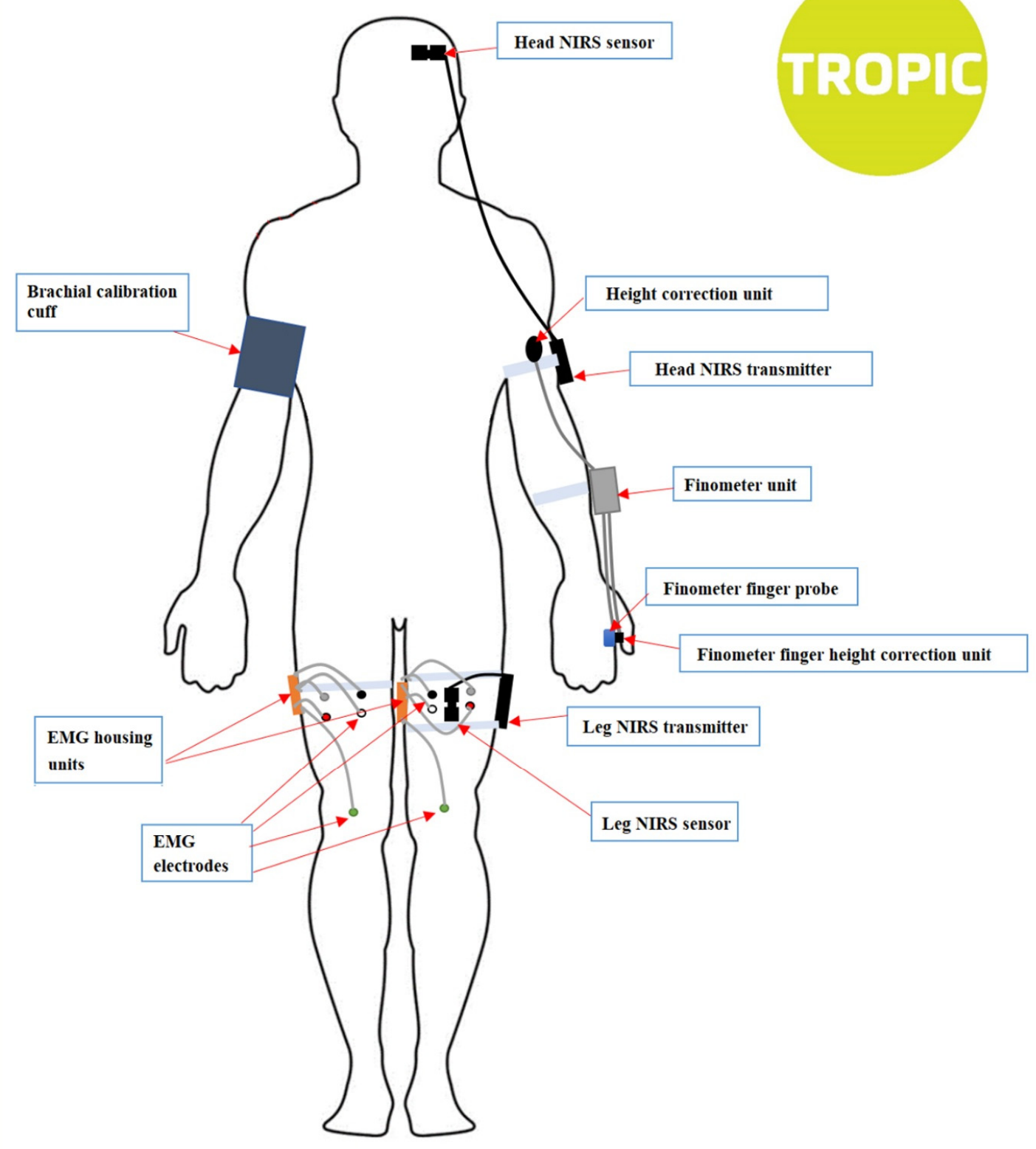

Figure 2. Sensor attachment diagram for the NCVI/NMFI assessment. A total of 6 modules with 15 sensors were attached to each of the participants.

\subsubsection{Head-Up Tilt}

A head-up tilt test is a simple, non-invasive examination that can be useful for the differential diagnosis of syncope and orthostatic intolerance syndromes [30,31]. In the head-up tilt test, unlike the active stand test, the motion of the tilt table provides the means 
of orthostatic challenge, with little muscle activation taking place. For the tilt procedure, which was medically supervised and started after a brief $(<5 \mathrm{~min})$ break following active stand, participants were affixed to the electrically motorized tilt table with footboard support. Approximately $10 \mathrm{~s}$ of travel time took place from 0 to $70^{\circ}$. The tilt table equipment was the same as for the active stand test, which is powered by a single-speed electric motor controlled by a pneumatic foot switch. During the tilt, participants were secured with two straps, fastened at the levels of the knees and chest. Throughout the tilt, participants underwent cardiovascular monitoring during an initial period of uninterrupted supine rest of at least $5 \mathrm{~min}$ and a subsequent head-up tilt to $70^{\circ}$ for $10 \mathrm{~min}$ or until symptoms developed.

\subsection{Instrumentation}

As well as the continuous non-invasive digital artery photoplethysmography for the extraction of cardiovascular signals, we also used NIRS monitoring for the extraction of regional tissue oxygenation in brain and muscle, and surface EMG to measure the muscle activation of the thigh muscles, both non-prompted and prompted (the latter in the form of thigh muscle squeezes that were specifically programmed into the assessment protocol). With a total of 6 modules and 15 sensors across Finapres ${ }^{\circledR}$ NOVA, NIRS and EMG, the attachment locations of the sensors and the placement of the transmitters (shown in Figure 2) were kept consistent for all participants by strict application of a Standard Operating Procedure, on which all researchers were trained.

\subsubsection{Finapres ${ }^{\circledR}$ NOVA}

The Finapres ${ }^{\circledR}$ NOVA, or NOVA for short, was used in this study to measure the finger blood pressure noninvasively on a beat-to-beat basis. The NOVA measures brachial blood pressure on command, from which corrections to the finger blood pressure are made (these oscillometric blood pressure measurements took place on the right arm during the calibration phases). The device also corrects for the hydrostatic height of the finger with respect to the heart level. The beat-to-beat data collected were extracted with the NOVAScope ${ }^{\circledR}$ v1.9 software.

\subsubsection{NIRS}

NIRS provides non-invasive and non-ionizing technology that has been used to measure variations in oxygenated and deoxygenated hemoglobin concentrations in various human tissues [32-34]. It has been shown that NIRS provides consistent readings with other measurement modalities in various applications, including cerebral blood flow [35] and skeletal muscle contractions [36]. With the time-resolved, frequency-domain, and continuous wave spectroscopic implementations of NIRS that have been developed in recent years, each with its own advantages and targeted applications [37], NIRS has a potential to be widely used in both research and clinical settings due to its versatility and high temporal resolution [38].

NIRS measurements record absorbance of light at several wavelengths, where changes in absorbance in the region near $850 \mathrm{~nm}$ are ascribed to oxygenated hemoglobin $(\mathrm{O} 2 \mathrm{Hb})$, and absorbance in the region near $760 \mathrm{~nm}$ is attributed to deoxygenated hemoglobin $(\mathrm{HHb})$ [39]. Combinations of the $\mathrm{O} 2 \mathrm{Hb}$ and $\mathrm{HHb}$ are often reported to describe physiological events, including oxygen saturation $(\mathrm{O} 2 \mathrm{Hb} /(\mathrm{O} 2 \mathrm{Hb}+\mathrm{HHb}))$ [40], hemoglobin difference $(\mathrm{O} 2 \mathrm{Hb}-\mathrm{HHb})$ [41], and tissue saturation index (TSI) [42].

Many commercial and custom-built NIRS instruments may be found in the market. These systems vary in terms of their application and system engineering, with trade-offs between light sources, detectors, and instrument electronics. In our study, we used the PortaLite $^{\circledR}$ (Artinis Medical Systems, The Netherlands), which is a wireless NIRS device capable of transmitting testing data through Bluetooth at a maximum sampling frequency of $50 \mathrm{~Hz}$. Oxysoft v3.2.70 was used as a user's interface for the setup, recording and exporting of NIRS data. The sampling frequency was set at $50 \mathrm{~Hz}$ for all participants. Two identical PortaLite ${ }^{\circledR}$ devices were used in the assessment setup, as shown in Figure 2, for 
measuring the oxygenation of the brain (left frontal lobe) and the left thigh (biceps femoris longus), respectively.

\subsubsection{Surface EMG}

EMG is a form of electrodiagnostic testing that measures muscle response or electrical activity in response to a nerve's stimulation of the muscle [43]. An electrodiagnostic signal examines the electrical currents generated in muscles during their contractions, which are interpreted as neuromuscular activity [44,45]. The invasive intramuscular EMG, which uses needles or fine wires as a primary means for signal acquisition, is traditionally prevalent in clinical settings [46]. Surface EMG is, on the other hand, a non-invasive technique for detecting, recording, and interpreting the electrical activity of groups of muscles at rest and during action [47-49]. In its simplest sense, surface EMG is a highly sensitive voltmeter that detects depolarization and hyperpolarization occurring on the sarcolemma, which are necessary for, and precede, the contraction of a muscle [50]. The usage of surface EMG is primarily applied to research environments, with few applications found in clinical laboratories, despite its clear potential as a non-invasive measure of muscle activity [51]. It is often considered more complex to analyze than intramuscular EMG as parameters of direct clinical relevance cannot be readily extracted from the recorded signal [52].

The SHIMMER ${ }^{\circledR}$ (Shimmer Sensing, Dublin, Ireland) is a wearable wireless sensing device that can be tailored to various research and industrial applications, including EMG, electrocardiography (ECG), and accelerometers. In the setting for this research, two SHIMMER ${ }^{\circledR}$ devices were used to monitor and record EMG signals for the rectus femoris and vastus lateralis in both legs. The sampling frequency was set to $1024 \mathrm{~Hz}$ and kept consistent for all participants. Just before starting the uninterrupted 5 min rest phase in both active stand and tilt, and after active stand and tilt, participants were asked to perform a 10-second maximum bilateral quadriceps contraction with continuous EMG and muscle NIRS monitoring.

\subsection{Data Management}

\subsubsection{Data Collection}

All data were collected immediately after each assessment. NOVA, NIRS, and EMG data were recorded and saved in time series format on three separate research computers. The beat-to-beat NOVA signals were recorded in NOVAScope ${ }^{\circledR}$ v1.9 and exported in cSv format. The NOVAScope ${ }^{\circledR}$ output consists of separate data frames, including the "Basic Nova" (e.g., systolic blood pressure (SBP), diastolic blood pressure (DBP), and heart rate (HR)) and "Advanced hemodynamics" (e.g., Cardiac Output CO, etc.) files. Each data frame contains its own "Time" variable corresponding to the time lapse from the beginning of the recording. The exported NIRS data frame contains signals from the two PortaLite ${ }^{\circledR}$ devices and a sample number variable, from which the time variable was derived with a sampling frequency of $50 \mathrm{~Hz}$. The two EMG devices export their data files separately, with each data frame containing its own time variable.

\subsubsection{Data Processing}

The data from NOVA, NIRS, and EMG were saved in situ in separate research computers at the end of each recording. Without any pre-processing, these raw data were transferred securely to a local research server where data processing and visualization took place.

\section{Data Merging}

All the raw data collected was processed in $\mathrm{R}$ version 4.0.5 using RStudio 1.4.1106 (Boston, MA, USA). The entire dataset consisted of three main groups of time series data files, namely the NOVA, NIRS, and EMG. As a convention adopted for this study, the data merging process began with the NOVA data files, where data frames including "Basic Nova" and "Advanced hemodynamics" were merged into one data frame by the time 
variable associated with each data frame. Similarly, the EMG data recorded for the left and right thighs were merged into one data frame. With NOVA, NIRS, and EMG data neatly organized, all three data frames were merged into one by the "Time" variable present in each data frame, forming the full data frame containing 70 variables with over 2 million observations for each participant's assessment. A consistent naming convention was adopted for the columns during merging, which was particularly helpful in streamlining the data syncing process.

\section{Three-Stage Data Syncing}

Although the newly merged full data frame contained all the variables from the assessment, it could not be used straightaway for visualization without precise syncing procedures across the entire data frame. Hence, a bespoke data syncing procedure was developed that consisted of three processes: instrument-level data syncing, fully merged data syncing, and cross-group data syncing (Figure 3). As the diagram in Figure 4 shows, a timer was used for syncing signals between NOVA, NIRS, and EMG, by manually pushing a key on the keyboard of each computer upon a $5 \mathrm{~s}$ countdown approaching each event during the assessment.
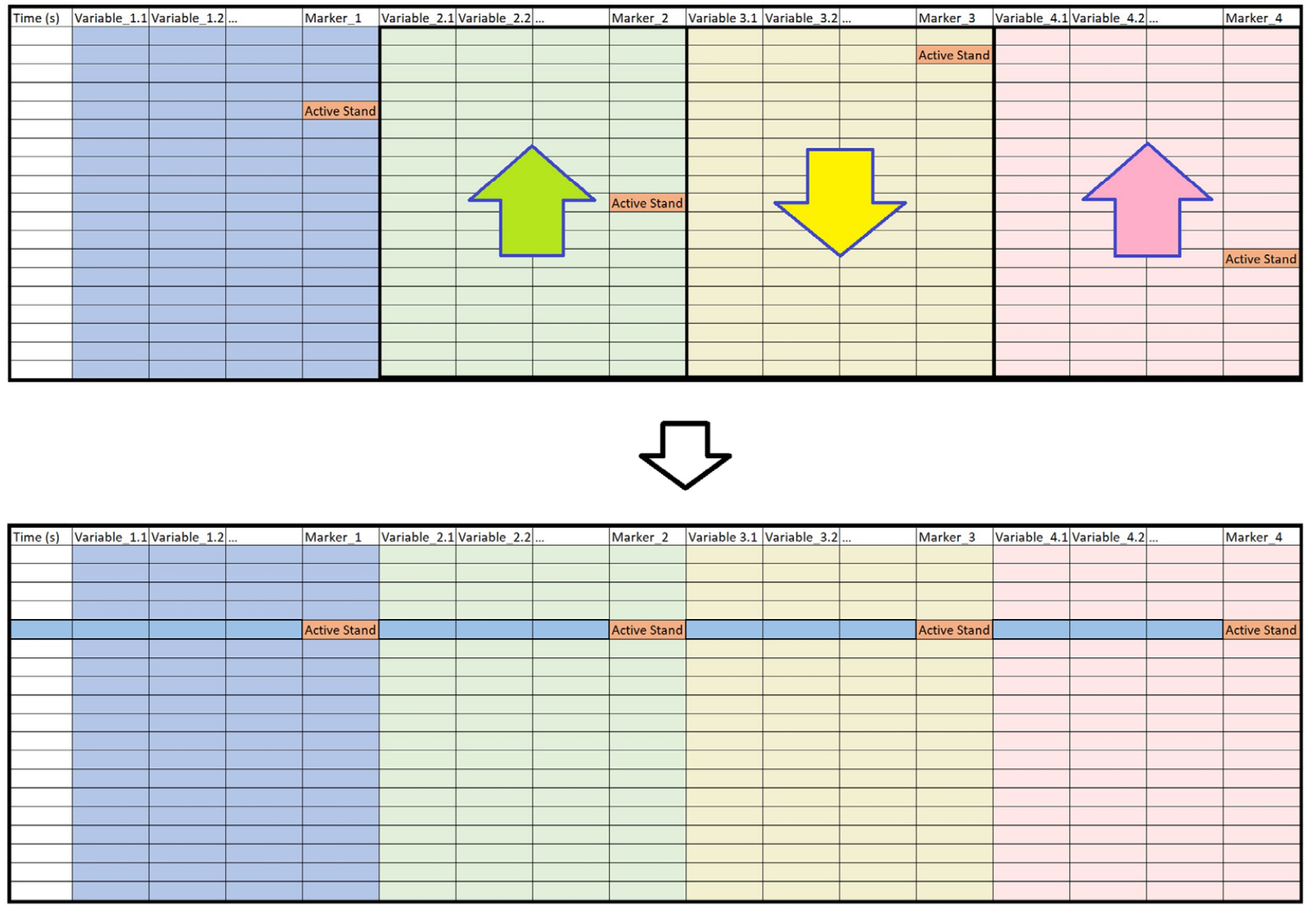

Figure 3. Illustration of the data syncing technique used in this study. A data frame that is merged from four separate time series data frames is shown on the top. There is an event marker variable "Active Stand" in each of the four constituent data frames, the time of which is located at different observations. The data frame is synced by shifting the variables in each of the constituent data frames, so that all the event marker variables are aligned, as shown at the bottom. 


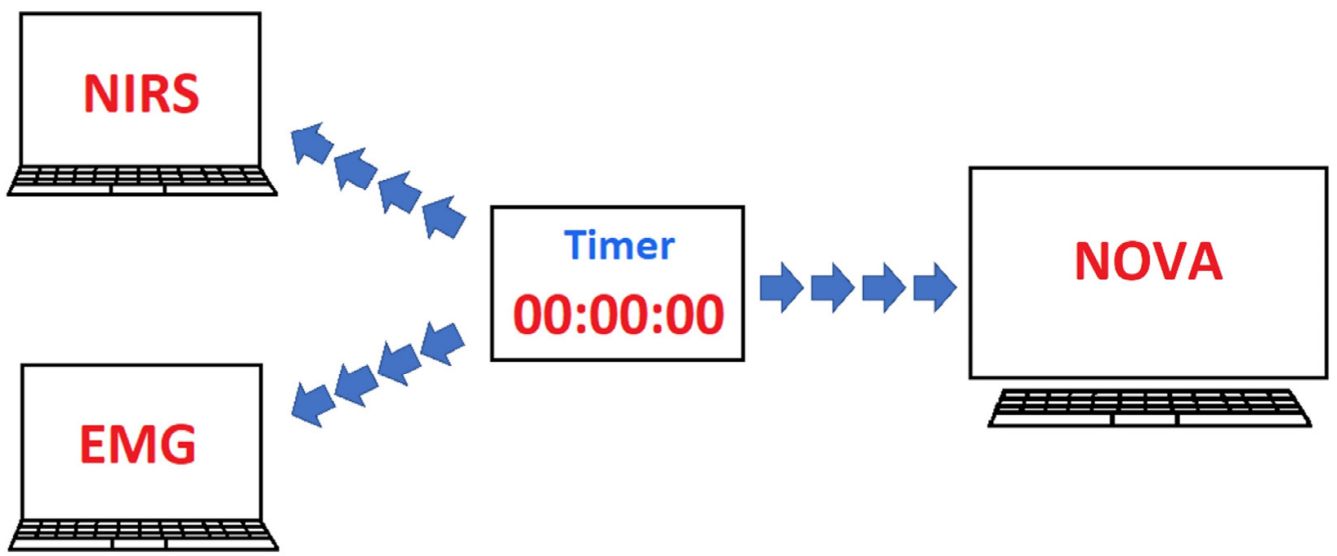

Figure 4. Communication diagram between different devices in the NCVI/NMFI setup. A timer was used for syncing signals between NOVA, NIRS, and EMG, by manually pushing a key on the keyboard of each computer upon a $5 \mathrm{~s}$ countdown approaching each event during the assessment.

\section{(1) Instrument-level data syncing}

As a first step, the NOVA data frame was synced. This was completed by shifting the columns that originated from each data file, so that all event markers appearing in the merged data frame were aligned, as illustrated in Figure 3 where, for illustration purposes, the event "Active Stand" is used for aligning the data frames. However, in practice, syncing could be done by any other event or moment in the assessment protocol. It is by the same method that the merged EMG data frames were synced.

(2) Full data frame syncing

With the device-level data synced, the columns that originated from NIRS and EMG data frames were adjusted, so that the "Active Stand" event markers across all constituent data were aligned within the final data frame. Due to the difference in sampling frequencies between NOVA, NIRS, and EMG signals, the final data frame contained missing values, which is of relevance for the subsequent visualization.

\section{(3) Cross-group data syncing}

After obtaining a fully synced data frame for each participant, it was then possible to perform cross-group comparison studies. Using the same technique, fully synced data frames of different participants can be merged and synced at any given time. Since it is often the case that the merged data frame is quite large at this stage, a good naming convention was key to organize the variables. If the variables of interest are already known, creating subsets of the full data frames prior to merging is advised.

\subsubsection{Visualization for Comparative Studies}

In addition to showcasing the capability of visualizing the raw signals of each participant's NCVI/NMFI assessment, we also aimed to demonstrate the practicality of the proposed methodology for comparative studies between participants. We arbitrarily selected the EMG data from two participants, including a participant feeling fatigued and a participant who did not report fatigue as part of the long COVID symptomatology. It should be noted that the purpose of the comparison was by no means to extract clinical conclusions on the effect of fatigue on physiological responses to the orthostatic challenges, but to exemplify the proposed data syncing and visualization technique.

\section{Results}

\subsection{Assessment Visualization}

With the bespoke data merging technique detailed above, a general overview of physiological behaviors during the NCVI/NMFI assessment can be efficiently visualized within the same plot, as exemplified in Figure 5 for the assessment of a participant who 
had been experiencing periodic postural orthostatic tachycardia symptoms. In this case, we chose to visualize (from top to bottom) EMG (left leg: channels 1 and 2), TSI of left leg and left frontal lobe, and SBP, DBP, CO, and HR. With cross-station signals fully integrated and precisely synced, possible interactions between different physiological signals in response to orthostatic stresses can be evaluated. Thanks to the addition of EMG signals, we were able to evidence the dynamics between muscle contractions of the thigh (both prompted and non-prompted) and neurocardiovascular responses throughout the assessment.

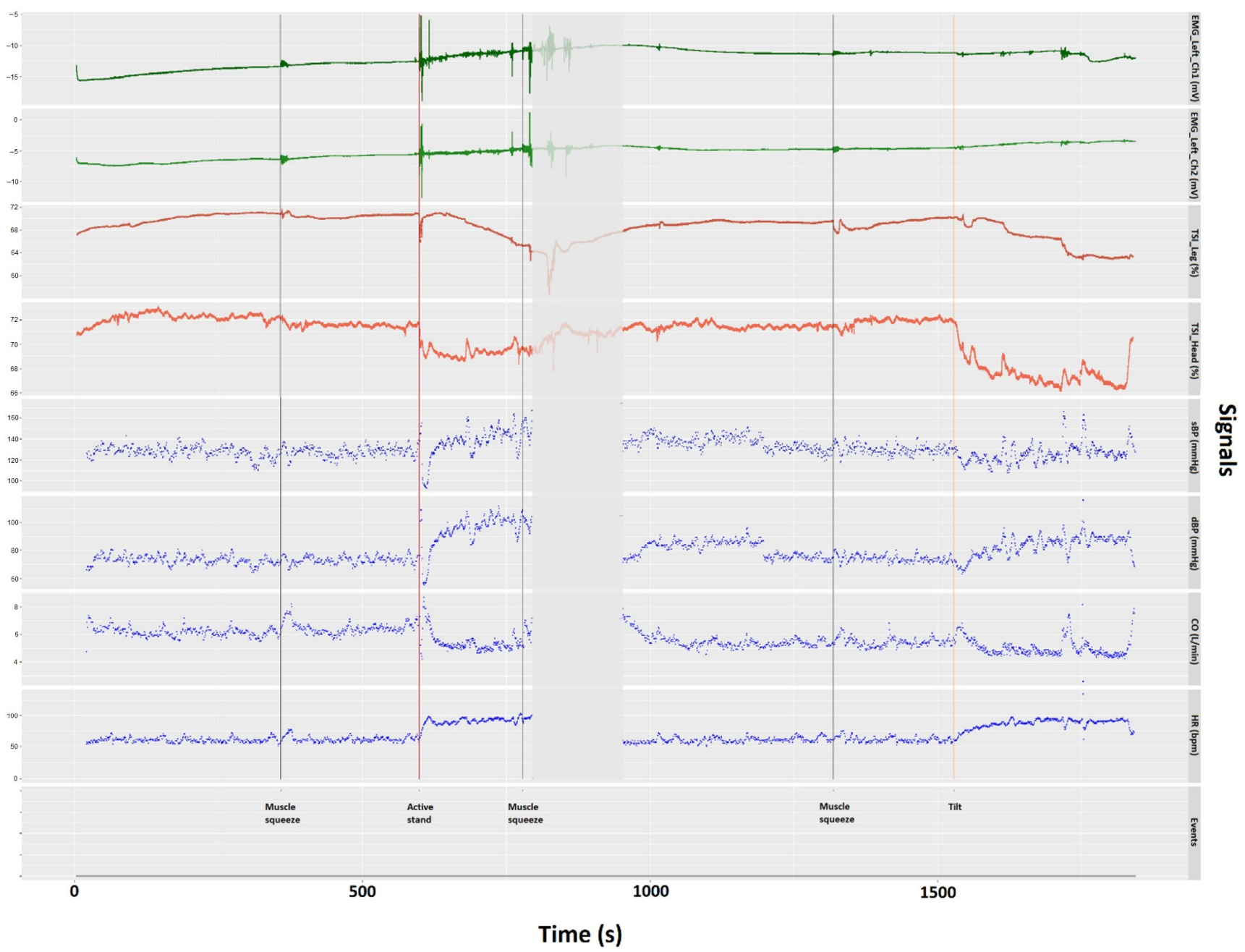

Figure 5. Visualization of integrated signals recorded from different instruments used in the NCVI/NMFI assessment, including the EMG amplitude of the left vastus lateralis (EMG_Left_Ch1) and rectus femoris (EMG_Left_Ch2), the TSI of the left thigh (TSI_Leg) and the left forehead (TSI_Head), the systolic blood pressure (SBP), the diastolic blood pressure (DBP), the cardiac output (CO), and the heart rate (shown as HR) of a participant. The vertical lines indicate the time at which all events took place, including active stand, head-up tilt, and prompted thigh muscle squeezes, annotated at the bottom of the plot.

As soon as the active stand took place (red vertical line in Figure 5), the participant experienced a transient (initial) drop in both SBP and DBP, which seemed to overshoot baseline readings afterwards. Derived cardiac output data showed the opposite trends. The heart rate increased immediately after the participant stood up before reaching a maximum of approximately $100 \mathrm{bpm}$ within $20 \mathrm{~s}$. This orthostatic tachycardia persisted after the participant recovered the baseline blood pressure levels. As indicated by the EMG green lines, muscle activation was evident during the pre-active stand "squeeze" and throughout the active standing phase, potentially reflecting changes in muscle sympathetic nerve 
activity [53,54]. Post-active stand, there seemed to be a sudden reduction in left frontal lobe TSI and a gradual reduction in left leg TSI.

As indicated by the breaks in blood pressure and heart rate signals between active stand and head-up tilt (shown as the greyed-out area in Figure 5), the beat-to-beat NOVA recording was paused for approximately $3 \mathrm{~min}$ to mitigate the discomfort felt by the participant's finger whilst leaving the finger cuff mounted and ready for the tilt phase.

Post tilt, the participant experienced a gradual increase in heart rate, which was sustained above baseline values until the test had to be prematurely terminated given ongoing symptoms of orthostatic intolerance. As the green EMG lines show, during the tilt there were a few non-prompted muscle contractions of the thigh muscles. Post-tilt there also seemed to be a sudden reduction in left frontal lobe TSI and a more gradual reduction in left leg TSI, possibly with more sudden "dips" in the latter associated with non-prompted contractions of the thigh muscles. Apparent peaks in CO and SBP were also suggested at the time of muscle contractions. In terms of SBP and DBP behavior during tilt, they both seemed to have increased variability with a possible overshoot in the latter.

\subsection{EMG Comparison}

For exemplification purpose, the EMG signals of a fatigued and a non-fatigued participant were compared by syncing at the onset of the active stand test, as shown in Figure 6 . Without any pre-processing, all four EMG signals shown in Figure 6 (two on each leg) contain noise and artefact of different origins. Generally, extrinsic sources that can be influenced by experimenters are responsible for most of the imperfections, including electrode selection, interelectrode distance and interfacial preparation between the electrodes and the skin [52,55]. It is also obvious that the baseline readings of the four EMG spectra are located in different amplitude ranges, which is likely to be caused by the difference in interelectrode distances that resulted from slight differences in electrode placement [56]. In addition, anatomical variances are an additional source of intrinsic noise, which may complicate inter-subject comparisons [52]. The effect of this noise and artefacts can be mitigated with different signal pre-processing techniques that are commonly applied to EMG spectra, including normalization, filtering, and segmentation [57]. Despite the noise and artefacts associated with the raw signals, these time series EMG spectra shown in Figure 6 can be adequately used for detecting the time of muscle contraction [58,59], both prompted and non-prompted, which was the original purpose of using surface EMG in this study.

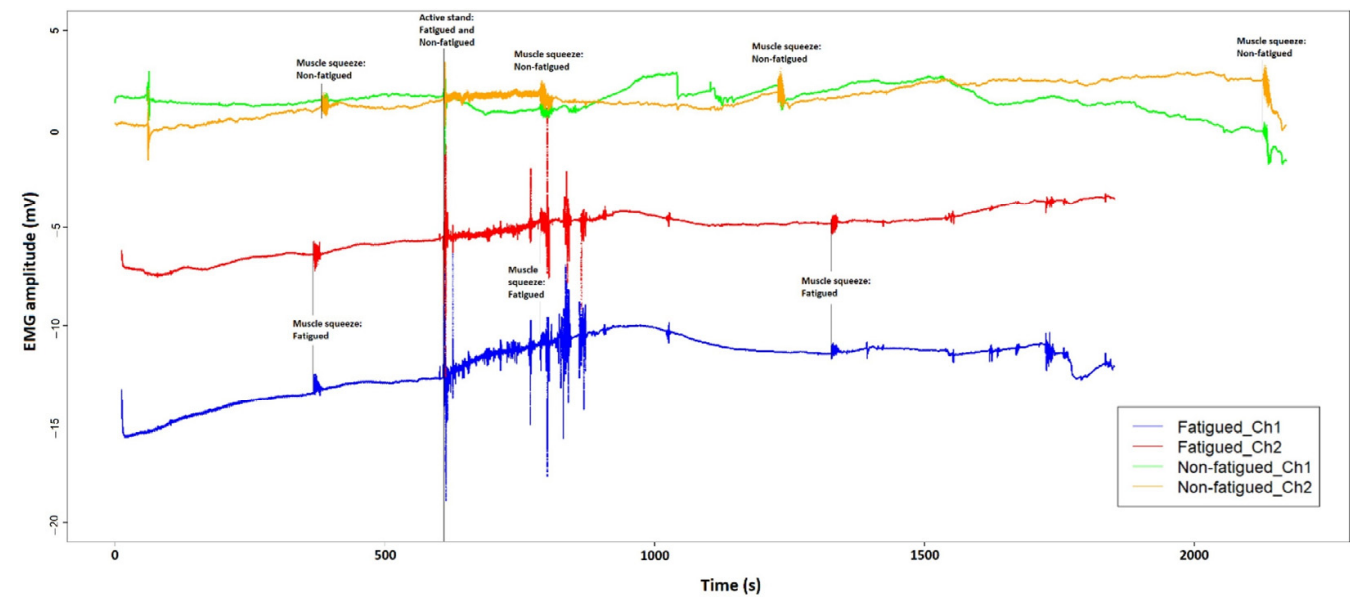

Figure 6. Surface EMG signals of the left vastus lateralis (shown as Ch1) and the left rectus femoris (shown as Ch2) of a fatigued participant, compared with a participant who did not report fatigue as part of the long COVID symptomatology. Thigh muscle contractions that were captured during the two recording sessions are marked by black vertical lines with corresponding annotations. The two data frames are synced at the onset of the active stand test at approximately $600 \mathrm{~s}$. 
Combining the epochs of the $10 \mathrm{~s}$ prompted contractions of the thigh reveals the characteristics of each active muscle squeeze performed during the assessment (see Figure 7, where the fourth contraction is not shown for the fatigued participant due to early tilt termination). Centered by a $10 \mathrm{~s}$ prompted muscle contraction, each epoch contains a $30 \mathrm{~s}$ unprocessed time series EMG recording. Although EMG signals without normalization may not be appropriate for cross-group comparisons, the amplitude of an EMG signal from a given muscle with the same electrode setup in the same recording session can be compared [60-62]. As suggested by Figure 7, the EMG amplitude in the fatigued participant seems to have a greater decline over time during the $10 \mathrm{~s}$ contraction, as well as showing greater signal artifact during the second squeeze. Even though the visualization in itself cannot be explanatory from a pathophysiological perspective, one may hypothesize from the visualization that the deterioration in the action potential in the fatigued participant over time could be an indication of underlying NMFI caused by a reduction in motor unit recruitment or firing rate [52].

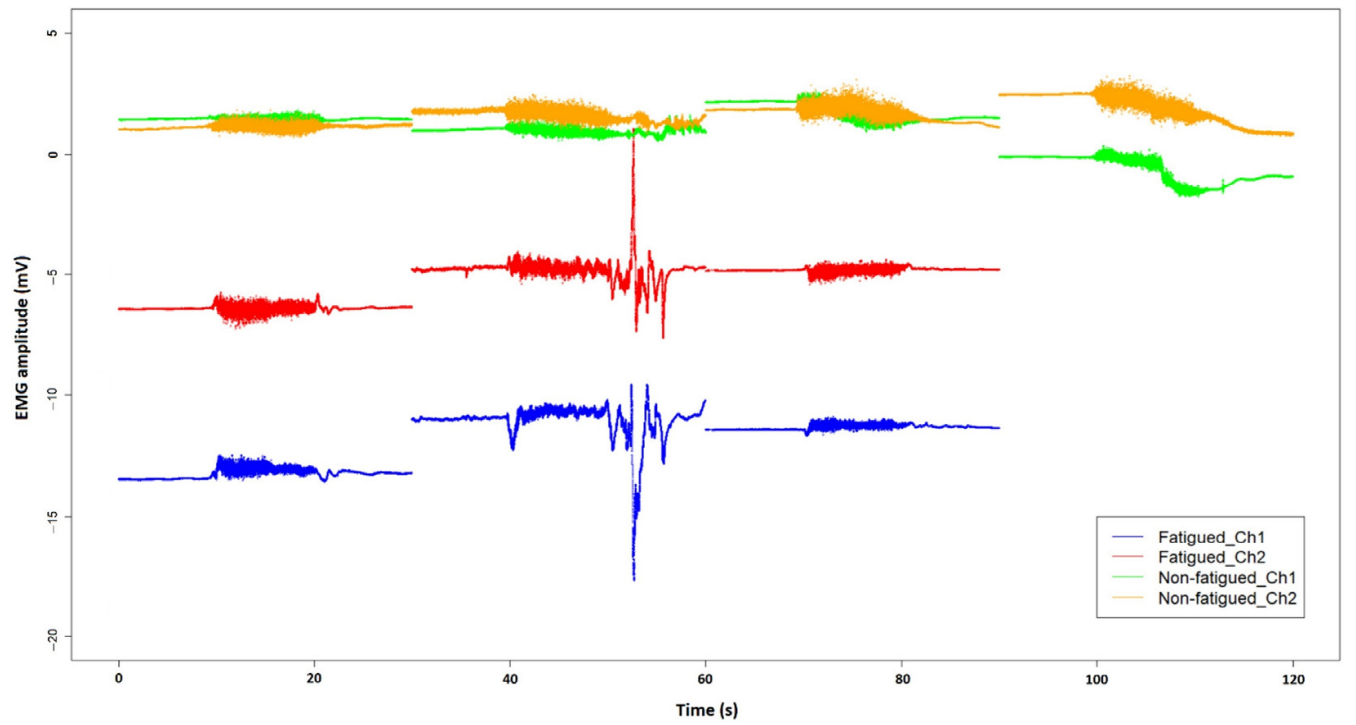

Figure 7. Surface EMG signals of the left vastus lateralis (shown as Ch1) and the left rectus femoris (shown as Ch2) of a fatigued participant, compared with those of a participant who did not report fatigue. An epoch of $30 \mathrm{~s}$ EMG signals is extracted for each $10 \mathrm{~s}$ prompted contraction of the thigh, together with $10 \mathrm{~s}$ before and after the muscle contraction. The fourth muscle squeeze for the fatigued participant was not performed due to early termination of the head-up tilt test.

\section{Discussion}

In this methodological study, we demonstrated that it is possible to visualize multiple independent physiological signals during a combined NCVI/NMFI assessment, which can capture the dynamics between skeletal muscle contractions and neurocardiovascular responses throughout the assessment. A proposed bespoke multimodal data visualization can offer an overview of the functioning of the muscle pump during both supine rest and orthostatic recovery and comparison studies can be conducted with signals from multiple participants at any given time in the assessment. This could help researchers and clinicians generate and test hypotheses based on the multimodal inspection of raw data in long COVID cohorts, where debilitating symptoms are common across multiple physiological systems [5,10]. In addition, the proposed methodology could provide insightful information in the assessment of various neurocardiovascular and neuromuscular conditions. Since the proposed data visualization technique is capable of integrating non-invasive signals from different sources at the greatest possible precision, it could potentially be used in a wide range of applications where a precise synchronization of cross platform signals is required. This could be particularly useful in the assessment of chronic systemic conditions that confer risk of both NCVI and NMFI, as seen, for example, in diabetes $[63,64]$. 


\subsection{Raw Data Visualization for NCVI/NMFI Assessment}

The proposed data merging and visualization technique was applied to the raw data directly exported from software packages for NOVA, NIRS, and EMG recordings. Without further processing, the final data frame captures a comprehensive set of information at the highest possible definition.

It has been the norm in many fields that raw data collected from assessments are pre-processed before visualization takes place, which increases processibility in data analysis [65], enhances the visual fidelity in presenting the results [66], and reduces the cost of storage and maintenance of the database [67]. However, one of the major disadvantages of data pre-processing is that it could lead to over-processing, thereby causing undesirable data loss that may be of pathophysiological and/or clinical relevance $[68,69]$. With raw data visualization, on the other hand, it is possible to integrate large data sets and visualize all information at the same time at the highest resolution and without losing any detail of the signals, thanks to the recent development of computational capabilities and software packages [70]. Indeed, our data visualization method was designed to show all relationships between signals in a summarized way. By also showing possible data artifacts, this visualization method defers to the researcher/clinician to comment on their possible physiological relevance, especially in new conditions such as long COVID where there is not much pre-existing medical literature. Indeed, we believe that a full raw data visualization approach is more appropriate than taking out or pre-filtering presumed artefactual signals from the outset, which is often completed by non-clinicians [71]. In addition, by "zooming out" the raw data visualization, transient signal artifacts are easier to detect (and sometimes lost to the naked eye), providing the clinician/researcher with a further insight as to the variability of the raw physiological signals at hand.

In the context of the NCVI/NMFI assessment, where so many physiological systems are expected to dynamically interact, we believe that the risks associated with not pre-processing the signals outweighs the benefit of data pre-processing in visualizing the NCVI/NMFI data, at least for hypothesis generation purposes. As demonstrated, the proposed methodology is sufficient for visualizing raw data from the multimodal NCVI/NMFI assessment without any need for signal pre-processing. We can seamlessly integrate participants' NOVA, NIRS, and EMG signals in a fully synced fashion, from which the relationship between different physiological responses could be visually examined for the purpose of generating pathophysiological hypotheses. Furthermore, our methodology also provides a platform where precisely synced assessment data can be conveyed to the next stage for data processing and bespoke analyses in specific applications, as desired. Given the fact that the interactions between muscle contraction and neurovascular physiological responses to orthostatic stressors are relatively understudied, the proposed raw data visualization methodology could pave the way for the further study of the role of the skeletal muscle pump in orthostatic intolerance syndromes and inform future clinical guidelines.

Despite the potential advantages of raw data visualization in NCVI/NMFI assessment, it requires moderately high computational assets to perform the tasks. Some coding skills in statistical software packages are also needed for processing the data. With the EMG recording at $1024 \mathrm{~Hz}$, the integrated data frames are quite large and therefore require a sizable secure storage space. To reduce the visualization processing time, an automation process is currently being implemented in the existing $R$ code. While it might not greatly shorten the running time for each participant, it would vastly improve the processing efficiency for the entire cohort.

\subsection{Data Syncing}

The merged data frames were synced by the events, which were manually marked by a timer with $5 \mathrm{~s}$ countdowns across three different instruments during each assessment. Despite every effort spent (as per Standard Operating Procedure) on the best possible synchronization of the key pushes, differences in reflex and human errors are inevitable at the operational level. While this issue could be potentially mitigated by scripting a 
single key push for marking events across all three sets of equipment, the integration of these systems could evoke complications in the clinical facility where the assessments took place. Hypothetically speaking, even if all three systems were integrated with seamlessly event calling commands, the signals written in the results files would not have been synced perfectly, which is the case for the manufacturer-supplied, supposedly fully synced NOVA data files. Despite having been validated and used in previous studies [72-74], the data files exported from the NOVA may have a discrepancy of as much as $2 \mathrm{~s}$ between .different signals. This is most likely an intrinsic issue caused by the modular architecture of both the hardware and software. With each component reporting its parameters to separate files, coupled with the fact that different calculation methods are used for the derived variables, the results files are written to the designated folders at different time stamps, as confirmed by the manufacturer. As a result, manual data syncing would still be needed before any processing and analysis could be done, regardless of the precision of syncing methods. Furthermore, integrating multiple Bluetooth devices with a slave configuration would very likely cause connection instability and random packet loss [75], which would further limit the permissible sampling frequency of its constituent devices. Therefore, we believe that it was appropriate to use the proposed manual syncing method, together with the precise data syncing procedures described in the present manuscript.

\subsection{EMG Sampling Frequency}

Bluetooth Low Energy (BLE), one of the most popular wireless data transmitting technologies, was used for both NIRS and EMG, thanks to its high data transmission rate, low power consumption, strong signal strength, miniaturized size, and low cost [76]. Due to the nature of Bluetooth connection, communication between the wearable modules and the host computer was limited to certain values, above which an unacceptable level of data loss can occur [77,78]. Therefore, the EMG data used in this study were extracted from the Secure Digital (SD) cards that were embedded into the SHIMMER ${ }^{\circledR}$ devices. However, due to the hardware architecture of these EMG devices, which use serial peripheral interface as the means for data communication between the microcontroller and the SD card, the characteristics of the data logging process set certain limits to the sampling frequency of the recorded analog signal to avoid excess packet drops [79]. This leads to a trade-off between sampling frequency, which dictates the details of the signals captured, and packet transmission, which governs the quality of the data received in the recording [80]. In general, high sampling frequencies tend to increase packet drops, which is a measure of data loss during the recording. It was discovered that setting the sampling frequency of EMG measurement to $512 \mathrm{~Hz}$ yielded a data loss of below 1\%. However, it is debatable whether $512 \mathrm{~Hz}$ could capture sufficient EMG information for physiological research [81]. It was also found that increasing the sampling frequency of the EMG recording to $2048 \mathrm{~Hz}$, which is recommended by previous studies [46,52], resulted in a packet drop of over $10 \%$, which is below the minimum required for EMG analysis, based on the manufacturer's manual. As a result, a sampling frequency of $1024 \mathrm{~Hz}$ was used for the EMG recording, which returned an acceptable data transfer rate of $96.5 \%$ according to the manufacturer's recommendations and was consistent with those used in previous investigations [82,83]. Despite meeting the minimum requirement for EMG recording based on the NyquistShannon Sampling Theorem, a sampling frequency of $1024 \mathrm{~Hz}$ is not optimal for capturing the exhaustive details of all muscle activities [46]. It is, however, appropriate for detecting muscle activation and amplitude monitoring [81], which were the sole usage of the EMG signals in this study.

\section{Conclusions}

A novel bespoke raw data visualization methodology was presented for combined NCVI/NMFI assessments of participants experiencing long COVID symptoms. This allowed functional visualization of both the autonomic nervous system and skeletal muscle pump in response to orthostatic stresses induced by the active stand and head-up tilt tests. 
We illustrated that signals from various instruments used in the NCVI/NMFI assessment, including NOVA, NIRS and EMG, can be seamlessly integrated and precisely synced. With the addition of EMG signals to the traditional NCVI assessment, we collected novel evidence that may help the understanding of the dynamics between muscle contractions of the thighs (both prompted and non-prompted) and neurocardiovascular responses throughout the assessment, which can potentially provide insights into the functioning of the skeletal muscle pump both during supine rest and in the recovery phase. Avenues for further refinement include the semi-automation of this method to reduce the visualization processing time and the subsequent dissemination of the proposed data visualization technique to a wide range of applications where precise cross-platform data syncing is required.

Author Contributions: Conceptualization: R.R.-O., A.M.; methodology: R.R.-O., A.M., F.X., G.J., clinical data collection: A.M., G.J., F.X., E.D., R.R.-O.; writing—original draft preparation: F.X., R.R.-O.; writing-review and editing: A.M., G.J., F.X., T.F., E.D., L.B., R.R.-O.; funding acquisition: R.R.-O.; resources: R.R.-O.; supervision: E.D., L.B., R.R.-O. All authors have read and agreed to the published version of the manuscript.

Funding: This study (Technology Assisted Solutions for the Recognition of Objective Physiological Indicators of Post-Coronavirus-19 Fatigue: TROPIC Study) was funded by a Grant from Science Foundation Ireland (SFI) under Grant number 20/COV/8493 and supported by SFI Grant number 18/FRL/6188. The funder had no role in the conduct of the research and/or preparation of the article; in study design; in the collection, analysis, and interpretation of data; in writing of the report; or in the decision to submit the paper for publication.

Institutional Review Board Statement: The study was conducted in accordance with the Declaration of Helsinki and received full approval by the St. James's Hospital/Tallaght University Hospital Joint Research Ethics Committee (Submission Number: 104: TROPIC; Approval Date: 4 May 2021) and the St. James's Hospital Research \& Innovation Office (Reference: 6566; Approval Date: 14 May 2021).

Informed Consent Statement: Written informed consent was obtained from all participants involved in the study.

Data Availability Statement: The data used in this manuscript are available upon request for verification purposes.

Acknowledgments: We are very grateful to our participants for their contribution and involvement in the study.

Conflicts of Interest: The authors declare no conflict of interest. The funders had no role in the design of the study; in the collection, analyses, or interpretation of data; in the writing of the manuscript, or in the decision to publish the results.

$\begin{array}{ll}\text { Abbreviations } \\ \text { OI } & \text { Orthostatic Intolerance } \\ \mathrm{NCVI} & \text { Neurocardiovascular Instability } \\ \mathrm{NMFI} & \text { Neuromuscular Function Impairment } \\ \mathrm{NIRS} & \text { Near-Infrared Spectroscopy } \\ \mathrm{EMG} & \text { Electromyography } \\ \mathrm{O}_{2} \mathrm{Hb} & \text { Oxygenated Hemoglobin } \\ \mathrm{HHb} & \text { Deoxygenated Hemoglobin } \\ \mathrm{TSI} & \text { Tissue Saturation Index } \\ \mathrm{SBP} & \text { Systolic Blood Pressure } \\ \mathrm{DBP} & \text { Diastolic Blood Pressure } \\ \mathrm{CO} & \text { Cardiac Output } \\ \mathrm{BLE} & \text { Bluetooth Low Energy }\end{array}$




\section{References}

1. WHO Coronavirus (COVID-19) Dashboard. 2022. Available online: https://covid19.who.int/ (accessed on 7 January 2022).

2. Ares-Blanco, S.; Astier-Peña, M.P.; Gómez-Bravo, R.; Fernández-García, M.; Bueno-Ortiz, J.M. The role of primary care during COVID-19 pandemic: A European overview. Aten Primaria 2021, 53, 102134. [CrossRef] [PubMed]

3. Lavie, G.; Weinstein, O.; Segal, Y.; Davidson, E. Adapting to change: Clalit's response to the COVID-19 pandemic. Isr. J. Health Policy Res. 2021, 10, 68. [CrossRef] [PubMed]

4. Lurie, N.; Saville, M.; Hatchett, R.; Halton, J. Developing Covid-19 vaccines at pandemic speed. N. Engl. J. Med. 2020, 382, 1969-1973. [CrossRef] [PubMed]

5. Jennings, G.; Monaghan, A.; Xue, F.; Mockler, D.; Romero-Ortuño, R. A Systematic Review of Persistent Symptoms and Residual Abnormal Functioning following Acute COVID-19: Ongoing Symptomatic Phase vs. Post-COVID-19 Syndrome. J. Clin. Med. 2021, 10, 5913. [CrossRef]

6. Maiese, A.; Frati, P.; Del Duca, F.; Santoro, P.; Manetti, A.C.; La Russa, R.; Di Paolo, M.; Turillazzi, E.; Fineschi, V. Myocardial pathology in COVID-19-associated cardiac injury: A systematic review. Diagnostics 2021, 11, 1647. [CrossRef]

7. Monaghan, A.; Jennings, G.; Xue, F.; Byrne, L.; Duggan, E.; Romero-Ortuno, R. Orthostatic intolerance in adults with long COVID was not associated with postural orthostatic tachycardia syndrome. Front. Physiol.-Auton. Neurosci. 2021, in press.

8. Dani, M.; Dirksen, A.; Taraborrelli, P.; Torocastro, M.; Panagopoulos, D.; Sutton, R.; Lim, P.B. Autonomic dysfunction in 'long COVID': Rationale, physiology and management strategies. Clin. Med. 2021, 21, e63-e67. [CrossRef]

9. Shouman, K.; Vanichkachorn, G.; Cheshire, W.P.; Suarez, M.D.; Shelly, S.; Lamotte, G.J.; Sandroni, P.; Benarroch, E.E.; Berini, S.E.; Cutsforth-Gregory, J.K.; et al. Autonomic dysfunction following COVID-19 infection: An early experience. Clin. Auton. Res. 2021, 31, 385-394. [CrossRef]

10. Lopez-Leon, S.; Wegman-Ostrosky, T.; Perelman, C.; Sepulveda, R.; Rebolledo, P.A.; Cuapio, A.; Villapol, S. More than 50 long-term effects of COVID-19: A systematic review and meta-analysis. Sci. Rep. 2021, 11, 16144. [CrossRef]

11. Paliwal, V.K.; Garg, R.K.; Gupta, A.; Tejan, N. Neuromuscular presentations in patients with COVID-19. Neurol. Sci. 2020, 41, 3039-3056. [CrossRef]

12. Guidon, A.C.; Amato, A.A. COVID-19 and neuromuscular disorders. Neurology 2020, 94, 959-969. [CrossRef] [PubMed]

13. O'Callaghan, S.; Kenny, R.A. Neurocardiovascular Instability and Cognition. Yale J. Biol. Med. 2016, 89, 59-71. [PubMed]

14. Collins, O.; Kenny, R.A. Is neurocardiovascular instability a risk factor for cognitive decline and/or dementia? The science to date. Rev. Clin. Gerontol. 2007, 17, 153-160. [CrossRef]

15. Finucane, C.; van Wijnen, V.K.; Fan, C.W.; Soraghan, C.; Byrne, L.; Westerhof, B.E.; Freeman, R.; Fedorowski, A.; Harms, M.P.M.; Wieling, W.; et al. A practical guide to active stand testing and analysis using continuous beat-to-beat non-invasive blood pressure monitoring. Clin. Auton. Res. 2019, 29, 427-441. [CrossRef] [PubMed]

16. Metzler, M.; Duerr, S.; Granata, R.; Krismer, F.; Robertson, D.; Wenning, G.K. Neurogenic orthostatic hypotension: Pathophysiology, evaluation, and management. J. Neurol. 2013, 260, 2212-2219. [CrossRef]

17. Tansey, E.A.; Montgomery, L.E.A.; Quinn, J.G.; Roe, S.M.; Johnson, C.D. Understanding basic vein physiology and venous blood pressure through simple physical assessments. Adv. Physiol. Educ. 2019, 43, 423-429. [CrossRef]

18. Stewart, J.M. Mechanisms of sympathetic regulation in orthostatic intolerance. J. Appl. Physiol. 2012, 113, 1659-1668. [CrossRef]

19. Biaggioni, I.; Shibao, C.A.; Diedrich, A.; Muldowney, J.A.S.; Laffer, C.L.; Jordan, J. Blood Pressure Management in Afferent Baroreflex Failure: JACC Review Topic of the Week. J. Am. Coll. Cardiol. 2019, 74, 2939-2947. [CrossRef]

20. Grubb, B. Neurocardiogenic syncope and related disorders of orthostatic intolerance. Circulation 2005, 111, 2997-3006. [CrossRef]

21. Masterson, M.M.; Morgan, A.L.; Multer, C.E.; Cipriani, D. The role of lower leg muscle activity in blood pressure maintenance of older adults. Clin. Kinesiol. 2006, 60, 8-17.

22. Garg, A.; Xu, D.; Blaber, A.P. Statistical validation of wavelet transform coherence method to assess the transfer of calf muscle activation to blood pressure during quiet standing. Biomed. Eng. Online 2013, 12, 132. [CrossRef] [PubMed]

23. Xu, D.; Verma, A.K.; Garg, A.; Bruner, M.; Fazel-Rezai, R.; Blaber, A.P.; Tavakolian, K. Significant role of the cardiopostural interaction in blood pressure regulation during standing. Am. J. Physiol. Heart Circ. Physiol. 2017, 313, H568-H577. [CrossRef] [PubMed]

24. Technology Assisted Solutions for the Recognition of Objective Physiological Indicators of Post-Coronavirus-19 Fatigue (TROPIC). Available online: https: / / clinicaltrials.gov/ct2/show / NCT05027724 (accessed on 7 January 2022).

25. Frith, J. Diagnosing orthostatic hypotension: A narrative review of the evidence. Br. Med. Bull. 2015, 2015. 115, 123-134. [CrossRef]

26. Fessel, J.; Robertson, D. Orthostatic hypertension: When pressor reflexes overcompensate. Nat. Clin. Pract. Nephrol. 2006, 2, 424-431. [CrossRef] [PubMed]

27. Fedorowski, A. Postural orthostatic tachycardia syndrome: Clinical presentation, aetiology and management. J. Intern. Med. 2019, 285, 352-366. [CrossRef] [PubMed]

28. Brignole, M.; Moya, A.; de Lange, F.J.; Deharo, J.C.; Elliott, P.M.; Fanciulli, A.; Feorowski, A.; Furlan, R.; Kenny, R.A.; Martin, A.; et al. 2018 ESC Guidelines for the diagnosis and management of syncope. Eur. Heart J. 2018, 39, 1883-1948. [CrossRef]

29. Wesseling, K.H. Finger arterial pressure measurement with Finapres. Z. Kardiol. 1996, 85, 38-44. [PubMed]

30. Teodorovich, N.; Swissa, M. Tilt table test today-State of the art. World J. Cardiol. 2016, 8, 277-282. [CrossRef]

31. Aponte-Becerra, L.; Novak, P. Tilt Test: A Review. J. Clin. Neurophysiol. 2021, 38, 279-286. [CrossRef] 
32. Ferrari, M.; Quaresima, V. A brief review on the history of human functional near-infrared spectroscopy (fNIRS) development and fields of application. Neuroimage 2012, 63, 921-935. [CrossRef]

33. Scholkmann, F.; Kleiser, S.; Metz, A.J.; Zimmermann, R.; Mata Pavia, J.; Wolf, U.; Wolf, M. A review on continuous wave functional near-infrared spectroscopy and imaging instrumentation and methodology. Neuroimage 2014, 85, 6-27. [CrossRef] [PubMed]

34. Cortese, L.; Zanoletti, M.; Karadeniz, U.; Pagliazzi, M.; Yaqub, M.A.; Busch, D.R.; Mesquida, J.; Durduran, T. Performance Assessment of a Commercial Continuous-Wave Near-Infrared Spectroscopy Tissue Oximeter for Suitability for Use in an International, Multi-Center Clinical Trial. Sensors 2021, 21, 6957. [CrossRef] [PubMed]

35. Pham, T.; Tgavalekos, K.; Sassaroli, A.; Blaney, G.; Fantini, S. Quantitative measurements of cerebral blood flow with near-infrared spectroscopy. Biomed. Opt. Express 2019, 10, 2117-2134. [CrossRef] [PubMed]

36. Ferrari, M.; Muthalib, M.; Quaresima, V. The use of near-infrared spectroscopy in understanding skeletal muscle physiology: Recent developments. Philos. Trans. R. Soc. A Math. Phys. Eng. Sci. 2011, 369, 4577-4590. [CrossRef]

37. Bunce, S.C.; Izzetoglu, M.; Izzetoglu, K.; Onaral, B.; Pourrezaei, K. Functional near-infrared spectroscopy. IEEE Eng. Med. Biol. Mag. 2006, 25, 54-62. [CrossRef] [PubMed]

38. Boas, D.A.; Elwell, C.E.; Ferrari, M.; Taga, G. Twenty years of functional near-infrared spectroscopy: Introduction for the special issue. Neuroimage 2014, 85, 1-5. [CrossRef] [PubMed]

39. Sanni, A.A.; McCully, K.K. Interpretation of Near-Infrared Spectroscopy (NIRS) Signals in Skeletal Muscle. J. Funct. Morphol. Kinesiol. 2019, 4, 28. [CrossRef]

40. Hesford, C.M.; Laing, S.J.; Cardinale, M.; Cooper, C.E. Asymmetry of quadriceps muscle oxygenation during elite short-track speed skating. Med. Sci. Sports Exerc. 2012, 44, 501-508. [CrossRef]

41. Tsuji, M.; duPlessis, A.; Taylor, G.; Crocker, R.; Volpe, J.J. Near infrared spectroscopy detects cerebral ischemia during hypotension in piglets. Pediatr. Res. 1998, 44, 591-595. [CrossRef]

42. Newman, L.; Nolan, H.; Carey, D.; Reilly, R.B.; Kenny, R.A. Age and sex differences in frontal lobe cerebral oxygenation in older adults-Normative values using novel, scalable technology: Findings from the Irish Longitudinal Study on Ageing (TILDA). Arch. Gerontol. Geriatr. 2020, 87, 103988. [CrossRef]

43. Elamvazuthi, I.; Zulkifli, Z.; Ali, Z.; Khan, M.K.A.A.; Parasuraman, S.; Balaji, M.; Chandrasekaran, M. Development of Electromyography Signal Signature for Forearm Muscle. Procedia Comput. Sci. 2015, 76, 229-234. [CrossRef]

44. Merletti, R.; Lo Conte, L.R. Surface EMG signal processing during isometric contractions. J. Electromyogr. Kinesiol. 1997, 7, 241-250. [CrossRef]

45. Raez, M.B.; Hussain, M.S.; Mohd-Yasin, F. Techniques of EMG signal analysis: Detection, processing, classification and applications. Biol. Proced. Online 2006, 8, 11-35. [CrossRef] [PubMed]

46. Merletti, R.; Cerone, G.L. Tutorial. Surface EMG detection, conditioning and pre-processing: Best practices. J. Electromyogr. Kinesiol. 2020, 54, 102440. [CrossRef] [PubMed]

47. Klasser, G.D.; Okeson, J. The clinical usefulness of surface electromyography in the diagnosis and treatment of temporomandibular disorders. J. Am. Dent. Assoc. 2006, 137, 763-771. [CrossRef] [PubMed]

48. Andrade, A.S.; Gavião, M.B.; Derossi, M.; Gameiro, G.H. Electromyographic activity and thickness of masticatory muscles in children with unilateral posterior crossbite. Clin. Anat. 2009, 22, 200-206. [CrossRef] [PubMed]

49. Cecílio, F.A.; Regalo, S.C.; Palinkas, M.; Issa, J.P.; Siéssere, S.; Hallak, J.E.; Machado-de-Sausa, J.P.; Semprini, M. Ageing and surface EMG activity patterns of masticatory muscles. J. Oral Rehabil. 2010, 37, 248-255. [CrossRef]

50. Vigotsky, A.D.; Halperin, I.; Lehman, G.J.; Trajano, G.S.; Vieira, T.M. Interpreting Signal Amplitudes in Surface Electromyography Studies in Sport and Rehabilitation Sciences. Front. Physiol. 2017, 8, 985. [CrossRef]

51. Felici, F.; Del Vecchio, A. Surface Electromyography: What Limits Its Use in Exercise and Sport Physiology? Front. Neurol. 2020, 11, 578504. [CrossRef]

52. McManus, L.; De Vito, G.; Lowery, M.M. Analysis and Biophysics of Surface EMG for Physiotherapists and Kinesiologists: Toward a Common Language With Rehabilitation Engineers. Front. Neurol. 2020, 11, 576729. [CrossRef]

53. Mano, T. Muscle Sympathetic Nerve Activity in Blood Pressure Control Against Gravitational Stress. J. Cardiovasc. Pharmacol. 2001, 38, S7-S11. [CrossRef] [PubMed]

54. Ray, C.A.; Rea, R.F.; Clary, M.P.; Mark, A.L. Muscle sympathetic nerve responses to static leg exercise. J. Appl. Physiol. 1992, 73, 1523-1529. [CrossRef] [PubMed]

55. De Luca, C.J.; Gilmore, L.D.; Kuznetsov, M.; Roy, S.H. Filtering the surface EMG signal: Movement artifact and baseline noise contamination. J. Biomech. 2010, 43, 1573-1579. [CrossRef] [PubMed]

56. Afsharipour, B.; Soedirdjo, S.; Merletti, R. Two-dimensional surface EMG: The effects of electrode size, interelectrode distance and image truncation. Biomed. Signal Processing Control. 2019, 49, 298-307. [CrossRef]

57. Chowdhury, R.H.; Reaz, M.B.; Ali, M.A.; Bakar, A.A.; Chellappan, K.; Chang, T.G. Surface electromyography signal processing and classification techniques. Sensors 2013, 13, 12431-12466. [CrossRef]

58. Hodges, P.W.; Richardson, C.A. Relationship between limb movement speed and associated contraction of the trunk muscles. Ergonomics 1997, 40, 1220-1230. [CrossRef]

59. Di Fabio, R.P. Reliability of computerized surface electromyography for determining the onset of muscle activity. Phys. Ther. 1987, 67, 43-48. [CrossRef] 
60. Mathiassen, S.E.; Winkel, J.; Hägg, G.M. Normalization of surface EMG amplitude from the upper trapezius muscle in ergonomic studies-A review. J. Electromyogr. Kinesiol. 1995, 5, 197-226. [CrossRef]

61. Erdelyi, A.; Sihvonen, T.; Helin, P.; Hänninen, O. Shoulder strain in keyboard workers and its alleviation by arm supports Int. Arch. Occup. Environ. Health 1988, 60, 119-124. [CrossRef]

62. Granström, B.; Kvarnström, S.; Tiefenbacher, F. Electromyography as an aid in the prevention of excessive shoulder strain. Appl. Ergon. 1985, 16, 49-54. [CrossRef]

63. Sardu, C.; De Lucia, C.; Wallner, M.; Santulli, G. Diabetes Mellitus and Its Cardiovascular Complications: New Insights into an Old Disease. J. Diabetes Res. 2019, 2019, 1905194. [CrossRef] [PubMed]

64. Trierweiler, H.; Kisielewicz, G.; Jonasson, T.H.; Petterle, R.R.; Moreira, C.A.; Borba, V.Z.C. Sarcopenia: A chronic complication of type 2 diabetes mellitus. Diabetol. Metab. Syndr. 2018, 10, 25. [CrossRef] [PubMed]

65. Wu, X.; Zhu, X.; Wu, G.; Ding, W. Data Mining with Big Data. Knowledge and Data Engineering. IEEE Trans. Knowl. Data Eng. 2014, 26, 97-107.

66. Tammen, H.; Hess, R. Data Preprocessing, Visualization, and Statistical Analyses of Nontargeted Peptidomics Data from MALDI-MS. Methods Mol. Biol. 2018, 1719, 187-196.

67. Chang, J.-R.; Chen, Y.-S.; Lin, C.-K.; Cheng, M.-F. Advanced Data Mining of SSD Quality Based on FP-Growth Data Analysis Appl. Sci. 2021, 11, 1715. [CrossRef]

68. Zhiyi, T.; Zhicheng, C.; Yuequan, B.; Hui, L. Convolutional neural network-based data anomaly detection method using multiple information for structural health monitoring. Struct. Control. Health Monit. 2018, 26, e2296.

69. García, S.; Ramírez-Gallego, S.; Luengo, J.; Benítez, J.M.; Herrera, F. Big data preprocessing: Methods and prospects. Big Data Anal. 2016, 1, 9. [CrossRef]

70. Yang, C.; Huang, Q.; Li, Z.; Liu, K.; Hu, F. Big Data and cloud computing: Innovation opportunities and challenges. Int. J. Digit. Earth 2016, 10, 13-53. [CrossRef]

71. Gupta, S.; Gupta, A. Dealing with Noise Problem in Machine Learning Data-sets: A Systematic Review. Procedia Comput. Sci. 2019, 161, 466-474. [CrossRef]

72. Schutte, A.E.; Huisman, H.W.; van Rooyen, J.M.; Malan, N.T.; Schutte, R. Validation of the Finometer device for measurement of blood pressure in black women. J. Hum. Hypertens. 2004, 18, 79-84. [CrossRef]

73. Hakim, K.; Mole, J.; Mahajan, R. Comparison of blood pressure (BP) measurements using arm cuff and finometry during induction of anaesthesia: 3AP1-8. Eur. J. Anaesthesiol. EJA 2010, 27, 45. [CrossRef]

74. Carlson, D.J.; Dieberg, G.; McFarlane, J.R.; Smart, N.A. Blood pressure measurements in research: Suitability of auscultatory, beat-to-beat, and ambulatory blood pressure measurements. Blood Press Monit. 2019, 24, 18-23. [CrossRef] [PubMed]

75. González-Cañete, F.J.; Casilari, E. Consumption Analysis of Smartphone based Fall Detection Systems with Multiple External Wireless Sensors. Sensors 2020, 20, 622. [CrossRef] [PubMed]

76. Tipparaju, V.V.; Mallires, K.R.; Wang, D.; Tsow, F.; Xian, X. Mitigation of Data Packet Loss in Bluetooth Low Energy-Based Wearable Healthcare Ecosystem. Biosensors 2021, 11, 350. [CrossRef]

77. Tosi, J.; Taffoni, F.; Santacatterina, M.; Sannino, R.; Formica, D. Performance Evaluation of Bluetooth Low Energy: A Systematic Review. Sensors 2017, 17, 2898. [CrossRef]

78. Wu, T.; Wu, F.; Qiu, C.; Redouté, J.M.; Yuce, M.R. A Rigid-Flex Wearable Health Monitoring Sensor Patch for IoT-Connected Healthcare Applications. IEEE Internet Things J. 2020, 7, 6932-6945. [CrossRef]

79. Bravo-Zanoguera, M.; Cuevas-Gonzalez, D.; Reyna, M.A.; Garcia-Vazquez, J.P.; Avitia, R.L. Fabricating a Portable ECG Device Using AD823X Analog Front-End Microchips and Open-Source Development Validation. Sensors 2020, 20, 5962. [CrossRef]

80. Bulić, P.; Kojek, G.; Biasizzo, A. Data Transmission Efficiency in Bluetooth Low Energy Versions. Sensors 2019, 19, 3746. [CrossRef]

81. Larivière, C.; Delisle, A.; Plamondon, A. Plamondon, The effect of sampling frequency on EMG measures of occupational mechanical exposure. J. Electromyogr. Kinesiol. 2005, 15, 200-209. [CrossRef]

82. Chu, J.U.; Moon, I.; Mun, M.S. A real-time EMG pattern recognition system based on linear-nonlinear feature projection for a multifunction myoelectric hand. IEEE Trans. Biomed. Eng. 2006, 53, 2232-2239.

83. Phinyomark, A.; Nuidod, A.; Phukpattaranont, P.; Limsakul, C. Feature Extraction and Reduction of Wavelet Transform Coefficients for EMG Pattern Classification. Electron. Electr. Eng. 2012, 122, 27-32. [CrossRef] 